\title{
Correction: An update on the management of gout
}

In the article 'An update on the management of gout' (DTB 2018;56:9-12), the following statement is incorrect: 'Prophylaxis should be considered when starting ULT, and based on the evidence from two recent systematic reviews, BSR recommends colchicine $\mathbf{5 0 0 m g}$ once or twice daily for up to 6 months.'

The sentence should read: 'Prophylaxis should be considered when starting ULT, and based on the evidence from two recent systematic reviews, BSR recommends colchicine $\mathbf{0 . 5} \mathbf{m g}$ once or twice daily for up to 6 months.'

DOI: $10.1136 / d t b .2018 .1 .0578$ corr1 